\title{
Validation of the Persian version of the Positive Mental Health Scale
}

\author{
Azam Naghavi ${ }^{1}$, Tobias Teismann ${ }^{2}$, Zahra Asgari ${ }^{1}$, Razieh Eizadifard ${ }^{2}$ and Julia Brailovskaia ${ }^{2^{*}}$
}

\begin{abstract}
Background: Positive mental health (PMH) is a factor of far-reaching salutogenetic importance. The present study aimed at validating the Persian version of the Positive Mental Health Scale (PMH-Scale).

Methods: Reliability and validity of the Persian version of the PMH-Scale were established in an Iranian student sample $(N=573)$. Internal consistency, convergent and discriminant validity were investigated, and exploratory factor analysis was conducted. Furthermore, it was assessed how PMH scores moderate the association between depressive symptoms and suicide ideation/behavior.

Results: The Persian version of the PMH-Scale was shown to have a unidimensional structure with excellent internal consistency, as well as good convergent and divergent validity. PMH differentiated between participants with higher vs. lower suicide risk. Furthermore, PMH proved to moderate the association between depressive symptoms and suicide ideation/behavior.
\end{abstract}

Conclusions: The results suggest that the PMH-Scale is a brief, reliable, and valid measure of subjective and psychological well-being that can be used in Iranian student samples and research settings.

Keywords: Positive mental health, Resilience, Suicide ideation/behavior, Assessment

\section{Background}

Mental health has traditionally been defined as the absence of psychopathology [1]: Individuals were seen as either mentally ill or presumed to be mentally healthy. Meanwhile, it is widely recognized that positive mental health (PMH), i.e., a positive sense of well-being, as well as the capacity to enjoy life and deal with life's challenges (cf., [1]), and the absence of psychopathology are not the same. It is rather the case that elements of PMH and psychopathology can be present at the same time ("dual factor model of metal health", e.g., [2]); as such $\mathrm{PMH}$ and psychopathology are not opposite ends of a single continuum; rather they represent different but correlated axes [3]. In this view, both $\mathrm{PMH}$ and psychopathology are required for complete mental health

* Correspondence: julia.brailovskaia@rub.de

${ }^{2}$ Mental Health Research and Treatment Center, Department of Psychology, Ruhr-Universität Bochum, Massenbergstrasse 9-13, 44787 Bochum, Germany Full list of author information is available at the end of the article assessments and should be integrated in research and practice. In fact, various studies point to the fact that $\mathrm{PMH}$ is a stronger predictor for the course of mental disorder than markers of psychopathology $[4,5]$. Nevertheless, most studies in clinical psychology and psychiatry continue to exclusively focus on negative aspects of mental health. To overcome this deficiency, timeefficient measurement instruments for the assessment of $\mathrm{PMH}$ are needed that are equally suitable for use in research and clinical practice.

The Positive Mental Health Scale (PMH-Scale) is such a new, brief, time-efficient and frequently used measure to assess PMH. The scale contains nine items [6] that capture aspects of subjective well-being ("I enjoy my life"), as well as items that capture aspects of psychological well-being, such as environmental mastery ("I manage well to fulfill my needs") and self-acceptance ("I am in good physical and emotional condition"). The $\mathrm{PMH}-\mathrm{Scale}$ thus integrates facets from two traditions of 
well-being research: The hedonic tradition focusing on positive affect and life-satisfaction and the eudemonic tradition focusing on optimal functioning in everyday life $[1,7]$. In validation studies of the PMH-Scale a unidimensional structure, good to excellent internal consistency (Cronbach's alpha $=.82-.93$ ), good testretest reliability $(\geq .74)$ and scalar invariance across samples and over time were demonstrated in research from various countries such as Germany [6], China and Russia [8], Lithuania [9], Pakistan [10], France, Poland, Spain, Sweden, the U.K. and the U.S. [11]. In correlational analyses, the PMH-Scale was negatively associated with selfreport measures of depression, anxiety and stress and positively associated with measures of social support, subjective happiness, and life satisfaction $[6,8]$.

In longitudinal predictor studies, $\mathrm{PMH}$, as assessed with the PMH-Scale [6], was found to be of central importance for the remission of mental disorders in general [4] and anxiety disorders [12-14] as well as suicidal ideation [15] in particular. Furthermore, current level of $\mathrm{PMH}$ was shown to be a unique predictor not only of the level of future $\mathrm{PMH}$, but also of the level of future mental health problems [16]. In two recent studies, $\mathrm{PMH}$ was identified as a prospective predictor of lower psychological burden experienced by the COVID-19 situation in spring 2020 [17], as well as of greater adherence and acceptance of COVID-19 related restrictions [11]. Finally - and most extensively researched - PMH has been shown to moderate the association between various risk factors (e.g., depression, stress, perceived burdensomeness, entrapment, addictive symptoms) and suicide ideation [18-21]. Furthermore, PMH was found to buffer the association between suicide ideation and suicide attempts [22]. These moderating properties of PMH were shown both in cross-sectional (e.g., [19]) and in longitudinal studies $[18,22-24]$. In a first crosscultural comparison, Siegmann et al. [19] found PMH to buffer the effect of depressive symptoms on suicide ideation in German and Chinese students: Students who reported high levels of PMH showed no increase in suicide ideation even as depression levels increased. Taken together, positive mental health seems to be of extensive salutogenetic importance.

In order to facilitate cross-cultural studies, it is essential to have validated measurement tools in different languages [8-11]. The comparison of Western individualistic cultures with more collectivistic cultures, such as Iran, is of particular interest. With the aim of enabling comparative cross-cultural studies of the conditions and significance of $\mathrm{PMH}$, the present study aimed to examine the factor structure, psychometric properties, and construct validity of the Persian version of the $\mathrm{PMH}-$ Scale within an Iranian student sample. Furthermore, it was aimed to investigate, whether $\mathrm{PMH}$, as assessed by the Persian version of the PMH-Scale, buffers the impact of depression on suicide ideation/behavior and as such confers resilience against suicide ideation and behavior in a country with a cultural background different from that in Germany and China [19]. In order to establish comparability with previous studies on the PMH-Scale (e.g., $[6,8-10,19])$ and since suicide ideation/behavior is common in student samples [25], the validation of the Persian PMH-Scale was performed on a student sample.

\section{Methods}

\section{Participants and procedure}

The current study sample comprised 573 participants from Iran $(73.1 \%$ women; age in years: $M(S D)=$ 24.45 (6.65, range: $18-76)$. All participants were students (63\% undergraduates, 37\% graduate students) with the following subjects of study: $60.7 \%$ liberal arts, $23.6 \%$ engineering sciences, $7.2 \%$ medical science, $5.8 \%$ basic science. Most of the participants were either single (79.2\%) or married (19.9\%; see [26] for more details).

Data were collected between March 2020 and May 2020 via an online survey. Participants were recruited via participation invitations displayed at social media (i.e., groups on Instagram, WhatsApp, and Telegram). There were no specific requirements for participation that was voluntary and not compensated. The implementation of the present study was approved by the responsible Ethics Committee. All participants were properly instructed and provided their informed consent online. No data sets were excluded. There were no missing data. A priori conducted power analyses (G*Power program, version 3.1) showed that the sample size was sufficient for valid results (power $>.80, \alpha=.05$, effect size $f^{2}=0.15$ ).

\section{Measures \\ Positive Mental Health Scale}

(PMH-Scale; original version: [6]). The PMH-Scale assessed subjective and psychological aspects of wellbeing across nine items (e.g., "I feel that I am actually well equipped to deal with life and its difficulties") that are rated on a 4-point Likert-type scale $(0=$ do not agree, $1=$ tend to disagree, $2=$ tend to agree, $3=$ agree $)$. Higher scores indicate higher levels of PMH. Previous research reported a scale reliability of Cronbach's $\alpha=.930$ [6] for the German language PMH-Scale and $\alpha=.921$ [11] for the English language PMH-Scale.

Translation of the PMH-Scale into the Persian language In the present study, the PMH-Scale was translated into the Persian language by the customary translation-back-translation-modification procedure by three bilingual speakers of the Persian language and of 
the English language [27]: The first person translated the English language version of the PMH-Scale into the Persian language. Then, two independent persons translated the Persian language version back into the English language. The back-translations were compared with each other and with the English version by the first person. Considering the differences between the backtranslations and the English version, this person modified the Persian version of the PMH-Scale. Next, the two independent translators translated again the modified Persian language version back into the English language. The process required overall three rounds of translation and back-translation before the Persian language version was judged to be satisfactory by all three translators.

\section{Multidimensional Scale of Perceived Social Support}

(MSPSS; original version: [28]; Persian version: [29]). Perceived social support from family, friends and significant others was assessed with the MSPSS. This instrument consists of twelve items (e.g., "There is a special person who is around when I am in need") that are rated on a 7 -point Likert-type scale $(0=$ very strongly disagree, $6=$ very strongly agree; current scale reliability: $\alpha=.922$ ). The higher the sum score, the higher the perceived social support.

\section{Post-Traumatic Growth Inventory}

(PTGI; original version: [30]; Persian version: [31]). Growth after traumatic events was measured with the PTGI that includes 21 items (e.g., "I changed my priorities about what is important in life"). The items belong to overall five domains (i.e., social connections, new possibilities, perceived skills and resources, life's appreciation, spiritual beliefs) and are rated on a 6-point Likerttype scale $(0=$ not at all, $5=$ extremely; current scale reliability: $\alpha=.937$ ). Higher sum scores indicate higher post-traumatic growth.

\section{PTSD Checklist}

(PCL-5; original version: [32]; Persian version: [33]). The PCL-5 assessed symptoms of PTSD (i.e., intrusion, avoidance, cognitive and mood alteration, arousal and reactivity alteration) with 20 items (e.g., "In the past month, how much were you been bothered by: "Repeated, disturbing, and unwanted memories of the stressful experience?") that are rated on a 5-point Likerttype scale $(0=$ not at all, $4=$ extremely; current scale reliability: $\alpha=.939$ ). The higher the sum score, the higher the sypmtoms of PTSD.

\section{Patient Health Questionnaire}

(PHQ-9; original version: [34]; Persian version: [35]). Depressive symptoms over the past two weeks were measured with the PHQ-9. The nine items (e.g., "Little interest or pleasure in doing things") are rated on a 4point Likert-type scale $(0=$ not at all, $3=$ nearly every day; current scale reliability: $\alpha=.879)$. Higher sum scores indicate higher depressive symptpms.

\section{Suicide Behaviors Questionnaire-Revised}

(SBQ-R; original version: [36]; Persian version: [37]). The SBQ-R comprises four items assessing different aspects of suicidal ideation/behavior (lifetime suicide ideation, suicide plans, suicide attempts, 12-months suicide ideation, suicidal communication, and one's estimation of how likely a future suicide attempt might be). Each item utilizes a different Likert scale with a sum score of 18 points indicating the highest severity of suicidal behavior. A cut-off score of 7 or higher has been established as an indicator for greater suicide risk [36]. The current scale reliability of the SBQ-R was $\alpha=.802$.

\section{Statistical analyses}

Statistical analyses were conducted using the Statistical Package for the Social Sciences (SPSS 26) and the macro Process version 3.5 (www.processmacro.org/index.html, [38]). After descriptive analyses, an exploratory factor analysis (EFA) using principal component analysis (PCA; rotation method: varimax) on the nine items that assessed PMH was calculated (see [39-41]). This step allowed to exploratorily investigate whether the unidimensional structure of the PMH-Scale that was reported by studies from other countries (e.g., Germany [6]) can be replicated in the present Iranian sample. Next, to investigate the construct validity of the PMH-Scale, its associations with social support and post-traumatic growth (convergent validity), as well as with depressive symptoms, PTSD and suicide ideation/behavior (discriminant validity) were assessed by the calculation of zero-order bivariate correlation analyses. Group differences in $\mathrm{PMH}$ scores between participants with greater suicide risk (SBQ- $R \geq 7)$ and participants with lower suicide risk $(\mathrm{SBQ}-\mathrm{R}<7)$ were tested via a t-test.

Finally, a moderation analysis (Process: model 1) examined the relationship between depressive symptoms (predictor), PMH (moderator) and suicide ideation/behavior (outcome), controlling for age and gender as covariates because of the mostly female and relatively young composition of the present sample. Following Hayes [38], the moderation effect was assessed by the bootstrapping procedure (10.000 samples) that provides percentile bootstrap confidence intervals (CI 95\%).

\section{Results}

Table 1 shows the descriptive statistics of the investigated variables. 
Table 1 Descriptive statistics of the investigated variables and their correlation with positive mental health

\begin{tabular}{llll}
\hline & $\boldsymbol{M}(\boldsymbol{S D})$ & Min-Max & $\boldsymbol{r}$ \\
\hline PMH-Scale & $15.40(6.18)$ & $0-27$ & - \\
MSPSS & $44.85(16.69)$ & $0-72$ & $.460^{* *}$ \\
PTGI & $58.99(21.09)$ & $3-105$ & $.531^{* *}$ \\
PHQ-9 & $10.09(6.47)$ & $0-27$ & $-.622^{* *}$ \\
PCL-5 & $28.91(17.57)$ & $0-95$ & $-.489^{* *}$ \\
SBQ-R & $5.30(3.07)$ & $3-18$ & $-.451^{* *}$ \\
\hline
\end{tabular}

$N=573$, MSPSS Multidimensional Scale of Perceived Social Support, PCL-5 PTSD Checklist, PHQ-9 Patient Health Questionnaire, PMH-Scale Positive Mental Health Scale, PTGI Post-Traumatic Growth Inventory, SBQ-R Suicide Behaviors Questionnaire-Revised, $M$ Mean, SD Standard Deviation, Min Minimum, Max Maximum. ${ }^{* *} p<001$

\section{Factor analysis}

In accordance with previous research (e.g., [6]), the EFA (Kaiser-Meyer-Olkin: $\mathrm{KMO}=.922 ;$ Barlett's test of sphericity: $\left.\chi^{2}=2877.787, \mathrm{df}=36, p<.001\right)$ revealed a 1 factor structure of the PMH-Scale. The factor had an eigenvalue of 5.214 and explained $57.93 \%$ of the variance (cf., [41]). This finding confirmed the unidimensional structure of the $\mathrm{PMH}-\mathrm{Scale}$ reported by previous research (e.g., $[6,8])$.

\section{Scale properties}

Internal consistency was assessed using Cronbach's $\alpha$. In the current study, it was $\alpha=.905$. The mean interitem correlation was: $r_{\mathrm{mi}}=.517$ (range: .285 to .783). The item-total scale correlation ranged between $r_{\text {it }}=.426$ (Item 1 ) and $r_{\text {it }}=.813$ (Item 2 ). The respectively deletion of single items provided no significant improvement of the internal consistency. The reliability ranged between $\alpha=.885$ (deletion of Item 2) and $\alpha=.913$ (deletion of Item 1). The item difficulty ranged between $p_{\mathrm{m}}=45.1 \%$ (Item 1 ) and $p_{\mathrm{m}}=63.4 \%$ (Item 9).

\section{Construct validity}

As shown in Table 1, PMH was significantly positively correlated with social support and with post-traumatic growth (both: $p<.001$ ). Its correlation with depressive symptoms, PTSD and suicide-related outcomes was significantly negative (all: $p<.001$ ). Furthermore, participants with greater suicide risk (SBQ- $\geq 7 ; n=146$, $25.5 \%)$ and participants with lower suicide risk (SBQ$\mathrm{R}<7, n=427,74.5 \%)$ differ significantly in $\mathrm{PMH}$ scores: $\mathrm{SBQ}-\mathrm{R} \geq 7: M(S D)=11.22(6.27), \mathrm{SBQ}-\mathrm{R}<7: M(S D)=$ $16.83(5.47), \mathrm{t}(225)=9.627, p<.001$. These findings reveal the convergent and the discriminant validity of the Persian version of the PMH-Scale.

\section{Moderation analysis}

The moderation model turned out to be statistically significant, $R^{2}=.292, \quad F \quad(5,567)=38.227, \quad p<.001$. The significant interaction between depressive symptoms and $\mathrm{PMH}, \mathrm{b}=-.011, \mathrm{SE}=.003,95 \% \mathrm{CI}[-.017,-.006], \mathrm{t}=-$ $4.031, p<.001$, revealed that the relationship between depressive symptoms and suicide ideation/behavior was moderated by PMH. According to the simple slopes tests, the positive link between depressive symptoms and suicide ideation/behavior was significant for low level (one $S D$ below mean $=-6.182: \mathrm{b}=.189, \mathrm{SE}=.028,95 \%$ CI $[.133, .244], \mathrm{t}=6.673, p<.001)$ and medium level of $\mathrm{PMH}($ mean $=0: \mathrm{b}=.122, \mathrm{SE}=.025,95 \%$ CI $[.073, .171]$, $\mathrm{t}=4.897, p<.001)$. Notably, the link was stronger for low than for medium level of PMH. However, the link between depressive symptoms and suicide ideation/behavior was not significant for high level of PMH (one SD above mean $=6.182: \mathrm{b}=.055, \mathrm{SE}=.032,95 \% \mathrm{CI}[-.007$, $.116], \mathrm{t}=1.737, p=.083)$. Thus, PMH significantly moderated the association between depressive symptoms and suicide ideation/behavior. Specifically, the lower the $\mathrm{PMH}$ level, the closer the link between both variables. Figure 1 visualizes the moderation effect.

\section{Discussion}

In the present study, the reliability and construct validity as well as the postulated factor structure of the Persian version of the Positive Mental Health Scale (PMH-Scale) were investigated. In line with the original version of the $\mathrm{PMH}-$ Scale [6] and its various translated versions [8, 9, $11]$, the Persian version of the PMH-Scale had a unidimensional factor structure and an excellent internal consistency. These results confirm previous findings from other countries (e.g., $[6,11]$ that described the $\mathrm{PMH}-\mathrm{Scale}$ as a time-efficient instrument for the assessment of positive mental health. Moreover, they extend the research on PMH by providing a valid and reliable Persian language version of this instrument that can be applied in Iranian samples.

Construct validity of the PMH-Scale was supported by expected associations between the PMH-Scale and depressive symptoms, suicide ideation/behavior and social support (cf., [6, 19]). The finding on significant associations between $\mathrm{PMH}$ scores and trauma-related constructs (i.e., post-traumatic symptoms and posttraumatic growth) extends prior work on the $\mathrm{PMH}$ and complements findings from a Lithuanian study showing a negative association between $\mathrm{PMH}$, life stressors and adjustment disorder symptoms [9]. Taken together the present results underscore the relevance of $\mathrm{PMH}$ in relation to a broad spectrum of psychopathological symptoms. A possible reciprocal relationship between $\mathrm{PMH}$ and post-traumatic growth should be considered in more detail in future studies (cf., [42]).

PMH scores differentiated between participants with higher vs. lower suicide risk. Furthermore, PMH moderated the association between depressive symptoms and 


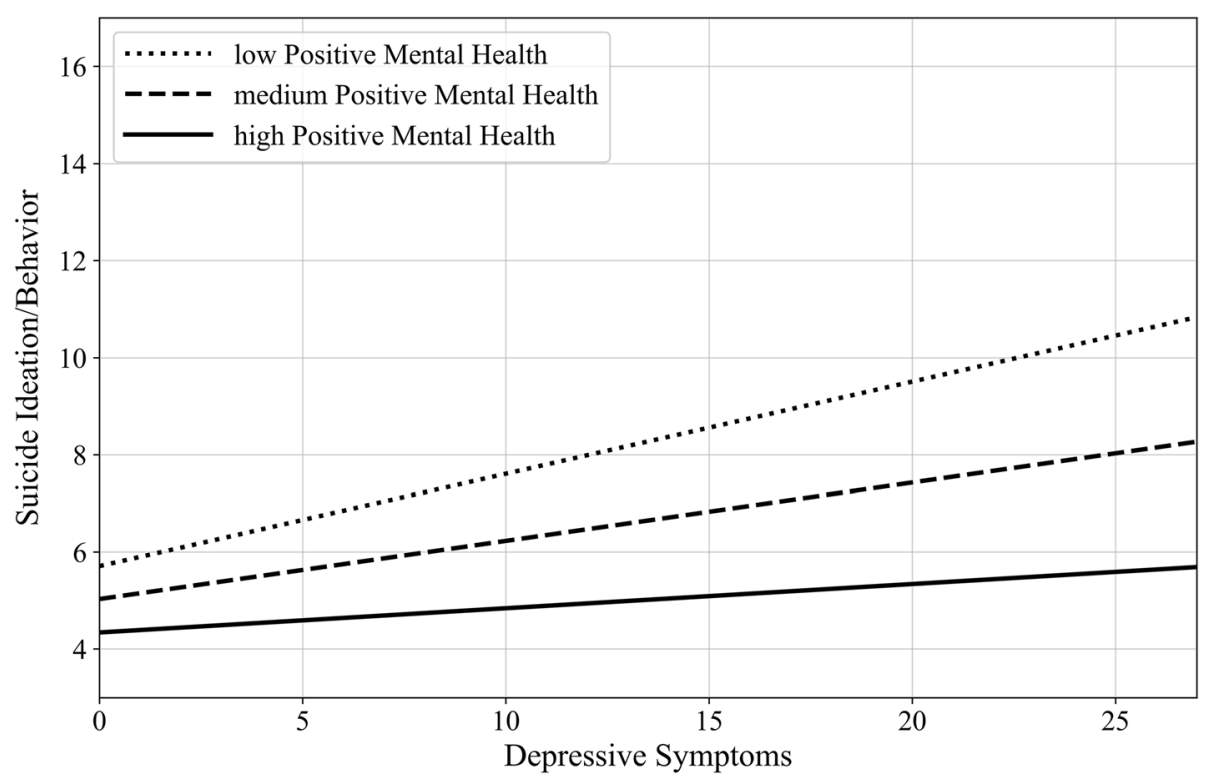

Fig. 1 Moderation effect of positive mental health (moderator) on depressive symptoms (predictor) to suicide ideation/behavior (outcome)

suicide ideation/behavior. Therefore, it was possible to replicate a finding previously shown in samples of German and Chinese students [19]: Those participants who reported a greater level of PMH were less likely to experience suicide ideation/behavior even at the highest severity of depressive symptoms as compared to participants who reported low level of PMH. This is yet another indication that $\mathrm{PMH}$ can be considered as conferring resilience (cf., [43]).

In terms of practical implications, the results of the current study underscore the importance of accounting for the presence of $\mathrm{PMH}$ in addition to aspects of psychopathology. Furthermore, the fact that PMH can significantly alter the impact of depressive symptoms on suicide ideation/behavior may be an important aspect to incorporate in clinical interventions. Finally, preventive programs for student populations may benefit from a focus on the fostering of PMH. Notably, first available studies showed that physical activity and loving kindness meditation have a positive impact on PMH [44, 45]. With respect to the fact that self-acceptance and environmental mastery are central facets of PMH [21], clinicians might also focus on fostering self-compassion [46] and (renewed) access to personal strengths and resources $[47,48]$.

Several limitations have to be considered when interpreting the current results. First, since $100 \%$ of the sample were students, it is unclear how the findings would generalize to a more diverse and/or clinical population. Yet, with regard both to suicide ideation/behavior [25] as well as changes in well-being [49] student populations are a group of special concern. Second, the cross- sectional design of the current study precludes analyses of test-retest reliability and scalar invariance over time. Longitudinal studies on these issues are warranted. In a similar vein, it is necessary to investigate, whether $\mathrm{PMH}$ does not only buffer the impact of depressive symptoms on suicide ideation/behavior in a cross-sectional study design, but also in a longitudinal study design (cf., [24]). Third, the current study utilized only self-report measures of depressive symptoms, post-traumatic symptoms, and suicide ideation/behavior. This method has certain advantages, for example, the measures are economical and easy to administer. However, self-report measures may fail to capture suicide ideation/behavior, depressive symptoms, or post-traumatic symptoms in their full complexity. Fourth, to establish convergent validity, it would have been good if other measures of $\mathrm{PMH}$, such as the Psychological Well-Being Scale [50] or the Satisfaction with Life Scale [51], had been available. Fifth, when investigating the factor structure of a scale, an exploratory factor analysis is recommended to be complemented by a confirmatory factor analysis. Both analyses should be calculated with independent samples [39-41]. In the present study, only one sample has been assessed that was used for the exploratory factor analysis. Therefore, future research is advised to further investigate the factor structure of the current Persian version of the $\mathrm{PMH}-\mathrm{Scale}$ by the calculation of a confirmatory factor analysis in a separate sample. Moreover, the current findings indicate that the cultural invariance of the Persian version of the PMH-Scale can be determined in a next step by the comparison of Iranian data with data gained in other countries [8, 42]. 


\section{Conclusion}

Despite some limitations, the current results suggest that the Persian version of the PMH-Scale is a brief, reliable, and valid measure of subjective and psychological wellbeing that can be used to complement mental health assessments in research and practice.

\section{Abbreviations}

MSPSS: Multidimensional Scale of Perceived Social Support; PCL-5: PTSD Checklist; PHQ-9: Patient Health Questionnaire; PMH: Positive Mental Health; PTGI: Posttraumatic Growth Inventory; SBQ-R: Suicide Behaviors

Questionnaire-revised

\section{Acknowledgements}

We acknowledge support by the Open Access Publication Funds of the Ruhr-Universität Bochum.

\section{Authors' contributions}

$A N, T, Z A, R E$ and JB originated the idea and contributed to data collection. $\Pi$ wrote the first manuscript draft. JB conducted the calculations and prepared Fig. 1. All authors contributed in the interpretation of the results and the writing and critical reviewing of the final manuscript. All authors read and approved the final manuscript.

\section{Funding}

Open Access Publication Funds of the Ruhr-Universität Bochum provided funding to pay the publication fees. Open Access funding enabled and organized by Projekt DEAL.

\section{Availability of data and materials}

All relevant data are reported within the paper. Analyzed data are available from the corresponding author on reasonable request.

\section{Declarations}

\section{Ethics approval and consent to participate}

All participants provided written informed consent. Furthermore, all procedures performed in studies involving human participants were in accordance with the ethical standards of the institutional and/or national research committee and with the 1964 Helsinki declaration and its later amendments or comparable ethical standards. The studies were approved by the University of Isfahan's Ethic Committee (IR.UI.REC.1399.008).

\section{Consent for publication}

All participants provided consent to publish all anonymized data reported in this publication.

\section{Competing interests}

All authors declare that they have no competing interest.

\section{Author details}

${ }^{1}$ Department of Counseling, Faculty of Education and Psychology, University of Isfahan, Azadi Sq, Isfahan 8174673441, Iran. ${ }^{2}$ Mental Health Research and Treatment Center, Department of Psychology, Ruhr-Universität Bochum, Massenbergstrasse 9-13, 44787 Bochum, Germany.

Received: 12 March 2021 Accepted: 15 September 2021

Published online: 27 September 2021

\section{References}

1. Keyes CLM. Mental illness and/or mental health? Investigating axioms of the complete state model of health. J Consult Clin Psychol. 2005;73(3):539-48. https://doi.org/10.1037/0022-006X.73.3.539.

2. Suldo SM, Shaffer EJ. Looking beyond psychopathology: the dual-factor model of mental health in youth. Sch Psychol Rev. 2008;1:52-68.

3. Trompetter HR, Lamers SMA, Westerhof GJ, Fledderus M, Bohlmeijer ET. Both positive mental health and psychopathology should be monitored in psychotherapy: confirmation for the dual-factor model in acceptance and commitment therapy. Behav Res Ther. 2017:91:58-63. https://doi.org/10.101 6/j.brat.2017.01.008.
4. Lukat J, Becker ES, Lavallee KL, van der Veld WM, Margraf J. Predictors of incidence, remission and relapse of axis I mental disorders in young women: a transdiagnostic approach. Clin Psychol Psychother. 2007;24:322-31.

5. Teismann T, Brailovskaia J, Siegmann P, Nyhuis P, Wolter M, Willutzki U. Dual factor model of mental health: co-occurence of positive mental health and suicide ideation in inpatients and outpatients. Psychiatry Res. 2018a;260: 343-5. https://doi.org/10.1016/j.psychres.2017.11.085.

6. Lukat J, Margraf J, Lutz R, van der Veld WM, Becker ES. Psychometric properties of the positive mental health scale (PMH-scale). BMC Psychol. 2016;4(1):8. https://doi.org/10.1186/s40359-016-0111-x.

7. Keyes CLM, Shmotkin D, Ryff CD. Optimizing well-being: the empirical encounter of two traditions. J Pers Soc Psychol. 2002;82(6):1007-22. https:// doi.org/10.1037/0022-3514.82.6.1007.

8. Bieda A, Hirschfeld G, Schönfeld P, Brailovskaia J, Zhang XC, Margraf J. Universal happiness? Cross-cultural measurement invariance of scales assessing positive mental health. Psychol Assess. 2017;29(4):408-21. https:// doi.org/10.1037/pas0000353.

9. Truskauskaite-Kuneviciene I, Kazlauskas E, Ostreikaite-Jurevice R, Brailovskaia J, Margraf J. Positive mental health and adjustment following life-stressors among young adults. Curr Psychol. 2020. https://doi.org/10.1007/s12144-02 0-00714-3.

10. Bibi A, Lin M, Margraf J. Salutogenic constructs across Pakistan and Germany: a cross sectional study. Int J Clin Health Psychol. 2020;20(1):1-9. https://doi.org/10.1016/j.jijchp.2019.10.001.

11. Margraf J, Brailovskaia J, Schneider S. Behavioral measures to fight COVID-19: an 8-country study of perceived usefulness, adherence and their predictors. PLoS One. 2020;15(12):e0243523. https://doi.org/10.1371/journal.pone.0243 523.

12. Teismann T, Brailovskaia J, Totzeck T, Wannemüller A, Margraf J. Predictors of remission from panic disorder, agoraphobia and specific phobia in outpatients receiving exposure therapy: the importance of positive mental health. Behav Res Ther. 2018b;108:40-4. https://doi.org/10.1016/j.brat.2018. 06.006.

13. Trumpf J, Becker ES, Vriends N, Meyer AH, Margraf J. Rates and predictors of remission in young woman with specific phobia. J Anxiety Disord. 2009; 23(7):958-64. https://doi.org/10.1016/j.janxdis.2009.06.005.

14. Vriends N, Becker E, Meyer A, Williams S, Lutz R, Margraf J. Recovery from social phobia in the community and its predictors. J Anxiety Disord. 2007; 21(3):320-37. https://doi.org/10.1016/j.janxdis.2006.06.005.

15. Teismann T, Forkmann T, Glaesmer H, Egeri L, Margraf J. Remission of suicidal thoughts: findings from a longitudinal epidemiological study. J Affect Disord. 2016;190:723-5. https://doi.org/10.1016/j.jad.2015.09.066.

16. Margraf J, Zhang XC, Lavallee K, Schneider S. Longitudinal prediction of positive and negative mental health in Germany, Russia, and China. PLoS One. 2020b;25(6):e0234997. https://doi.org/10.1371/journal.pone.0234997.

17. Brailovskaia J, Margraf J. Predicting adaptive and maladaptive responses to the coronavirus (COVID-19) outbreak: a prospective longitudinal study. Int J Clin Health Psychol. 2020;20(3):181-91. https://doi.org/10.1016/j.ijchp.2020. 06.002.

18. Brailovskaia J, Teismann T, Margraf J. Positive mental health mediates the relationship between Facebook addiction disorder and suicide-related outcomes: a longitudinal approach. Cyberpsych Beh Soc N. 2020;23(5):34650. https://doi.org/10.1089/cyber.2019.0563.

19. Siegmann P, Teismann T, Fritsch N, Forkmann T, Glaesmer H, Zhang XC, et al. Resilience to suicide ideation: a cross-cultural test of the buffering hypothesis. Clin Psychol Psychother. 2018;25(1):e1-9. https://doi.org/10.1 002/cpp. 2118

20. Siegmann P, Willutzki U, Fritsch N, Nyhuis P, Wolter M, Teismann T. Positive mental health as a moderator of the association between risk factors and suicide ideation/behavior in psychiatric inpatients. Psychiatry Res. 2019;273: 678-84. https://doi.org/10.1016/j.psychres.2019.01.091.

21. Teismann T, Brailovskaia J. Entrapment, positive psychological functioning and suicide ideation: a moderation analysis. Clin Psychol Psychother. 2020 27(1):34-41. https://doi.org/10.1002/cpp.2403.

22. Brailovskaia J, Forkmann T, Glaesmer H, Paashaus L, Rath D, Schönfelder A, et al. Positive mental health moderates the association between suicide ideation and suicide attempts. J Affect Disord. 2019;245:246-9. https://doi. org/10.1016/j.jad.2018.11.005.

23. Brailovskaia J, Teismann T, Zhang XC, Margraf J. Positive mental health, stressful life events and suicide ideation: a two-year follow-up study. Crisis. 2020;41(5):383-8. https://doi.org/10.1027/0227-5910/a000652. 
24. Teismann T, Forkmann T, Brailovskaia J, Siegmann P, Glaesmer H, Margraf J. Positive mental health moderates the association between depression and suicide ideation: a longitudinal study. Int J Clin Health Psychol. 2018c;18(1): 1-7. https://doi.org/10.1016/j.ijchp.2017.08.001.

25. Mortier P, Auerbach RP, Alonso J, Axinn WG, Cuijpers P, Ebert DD, et al. Suicidal thoughts and behaviors among college students and same-aged peers: results from the world health organization world mental health surveys. Soc Psychiatry Psychiatr Epidemiol. 2018;53(3):279-88. https://doi. org/10.1007/s00127-018-1481-6

26. Naghavi A, Teismann T, Asgari Z, Mohebbian MR, Mansourian M, Mañanas MÁ. Accurate diagnosis of suicide ideation/behavior using robust ensemble machine learning: a university student population in the Middle East and North Africa (MENA) region. Diagnostics. 2020;10:956.

27. Berry JW. Introduction to methodology, in: Triandis, H., Berry, J.W. (Eds.), handbook of cross-cultural psychology (pp. 1-28). Boston: Allyn \& Bacon; 1989.

28. Zimet GD, Dahlem NW, Zimet SG, Farley GK. The multidimensional scale of perceived social support. J Pers Assess. 1988;52(1):30-41. https://doi.org/1 0.1207/s15327752jpa5201_2.

29. Rostami R, Shahmohamadi K, Ghaedi G, Besharat MA, Akbari Zardkhaneh S, Nosratabadi M. Relations among self-efficacy, emotional intelligence and perceived social support in university students. Horizon Med Sci. 2010;16: 46-54.

30. Tedeschi RG, Calhoun LG. The posttraumatic growth inventory: measuring the positive legacy of trauma. J Trauma Stress. 1996;9(3):455-71. https://doi. org/10.1002/jts.2490090305.

31. Mahmoudi S, Rahimi C, Mohammadi N. Psychometric properties of posttraumatic growth inventory in an Iranian sample. J Psychol Models Meth. 2013;3:93-108.

32. Weathers FW, Litz B, Keane TM, Palmieri PA, Marx BP, Schnurr PP. The PTSD Checklist for dsm-5 (PCL-5). National Center for PTSD. 20. 2013; Retrieved from www.ptsd.va.gov (accessed on 12 March 2021).

33. Sadeghi M, Taghva A, Goudarzi N, Rah NA. Validity and reliability of persian version of "post-traumatic stress disorder scale" in war veterans. Iranian J War Pub Health. 2016;8:243-9.

34. Kroenke K, Spitzer RL. The PHQ-9: a new depression diagnostic and severity measure. Psychiatr Ann. 2002;32(9):509-15. https://doi.org/10.3928/00485713-20020901-06.

35. Ardestani MS, Ashtiani RD, Rezaei Z, Vasegh S, Gudarzi SS. Validation of Persian version of PHQ-9 for diagnosis of major depressive episode in psychiatric wards in Iran. Int J Appl Behav Sci. 2019;5:1-8.

36. Osman A, Bagge CL, Gutierrez PM, Konick LC, Kopper BA, Barrios FX. The suicidal behaviors questionnaire-revised (SBQ-R): validation with clinical and nonclinical samples. Assessment. 2001;8(4):443-54. https://doi.org/10.1177/1 07319110100800409

37. Amini-Tehrani M, Nasiri M, Jalali T, Sadeghi R, Ghotb, Zamania H. Validation and psychometric properties of Suicide Behaviors Questionnaire-Revised (SBQ-R) in Iran. Asian J Psychiatr. 2020;47:101856

38. Hayes A. Introduction to mediation, moderation, and conditional process analysis. 2nd ed. New York: The Guilford Press; 2021.

39. Field A. Discovering statistics using IBM SPSS statistics. London: Sage Publications Ltd.; 2013.

40. Bühner M. Einführung in die Test-und Fragebogenkonstruktion. München: Pearson Deutschland GmbH; 2011.

41. Schmitt TA. Current methodological considerations in exploratory and confirmatory factor analysis. J Psychoeduc Assess. 2011;29(4):304-21. https:// doi.org/10.1177/0734282911406653.

42. Bieda A, Hirschfeld G, Schönfeld P, Brailovskaia J, Lin M, Margraf J. Happiness, life satisfaction and positive mental health: investigating reciprocal effects over four years in a Chinese student sample. J Res Pers. 2019;78:198-209. https://doi.org/10.1016/j.jp.2018.11.012.

43. Johnson J, Wood AM, Gooding P, Taylor PJ. Tarrier. Resilience to suicidality: the buffering hypothesis. Clin. Psychol. Rev. 2011;31:563-91.

44. Totzeck C, Teismann T, Hofmann SG, Pflug V, von Brachel R, Zhang X, et al. Loving-kindness meditation promotes mental health in university students. Mindfulness. 2020;11(7):1623-31. https://doi.org/10.1007/s12 671-020-01375-w.

45. Velten J, Bieda A, Scholten S, Wannemüller A, Margraf J. Lifestyle choices and mental health: a longitudinal survey with German and Chinese students. BMC Public Health. 2018;18(1):632. https://doi.org/10.1186/s12889018-5526-2
46. Gilbert P. The compassionate mind. London: Robinson; 2010.

47. Willutzki U, Teismann T. Ressurcenaktivierung in der Psychotherapie. Göttingen: Hogrefe; 2013.

48. Fava G. Well-being therapy. Basel: Karger; 2016. https://doi.org/10.1159/isbn. 978-3-318-05822-2.

49. Brailovskaia J, Margraf J. Decrease of well-being and increase of online media use: Cohort trends in German university freshmen between 2016 and 2019. Psychiatry Res. 2020;290:113110.

50. Ryff CD. (1989). Happiness is everything, or is it? Explorations on the meaning of psychological well-being. J Pers Soc Psychol. 1989;57:1069-81.

51. Diener E, Emmons RA, Larsen RJ, Griffin S. The satisfaction with life scale. J Pers Assess. 1985;49(1):71-5. https://doi.org/10.1207/s15327752jpa4901_13.

\section{Publisher's Note}

Springer Nature remains neutral with regard to jurisdictional claims in published maps and institutional affiliations.
Ready to submit your research? Choose BMC and benefit from:

- fast, convenient online submission

- thorough peer review by experienced researchers in your field

- rapid publication on acceptance

- support for research data, including large and complex data types

- gold Open Access which fosters wider collaboration and increased citations

- maximum visibility for your research: over $100 \mathrm{M}$ website views per year

At BMC, research is always in progress.

Learn more biomedcentral.com/submissions 